\title{
Progression of diabetic nephropathy after successful pancreas transplantation alone: a case report
}

\author{
Yoonhong Kim', Dong Il Kim', Jae Ryong Shim ${ }^{1}$, Tae Beom Lee', Kwang Ho Yang ${ }^{1}$, Je Ho Ryu', \\ Hyun Jung Lee ${ }^{2}$, Byung Hyun Choi ${ }^{1}$ \\ Departments of ${ }^{1}$ Surgery and ${ }^{2}$ Pathology, Pusan National University Yangsan Hospital, \\ Pusan National University School of Medicine, Yangsan, Korea
}

\begin{abstract}
Pancreas transplantation is the only method that can nearly cure insulin-dependent diabetes mellitus. However, the effect of pancreas transplantation on patients with diabetic nephropathy has recently been considered controversial. In this report, we present a case of abrupt aggravation of proteinuria after successful pancreas transplantation alone without evidence of calcineurin inhibitor (CNI) toxicity. A 22-year-old female patient with type I diabetes mellitus underwent pancreas transplantation alone. The patient already had retinopathy and mild proteinuria, which in this case, may mean diabetic nephropathy. Her glucose levels were managed within the normal range after successful pancreas transplantation. However, the amount of proteinuria fluctuated. Kidney needle biopsy was performed owing to severe elevation of proteinuria, 2 years after the transplantation. Electron microscopy revealed diabetic glomerulosclerosis without evidence of CNI toxicity. This case indicates that diabetic nephropathy can be aggravated after pancreas transplantation, despite well-managed glucose levels and absence of CNI toxicity.
\end{abstract}

Keywords: Pancreas transplantation; Diabetic nephropathies; Calcineurin inhibitors

\section{INTRODUCTION}

Pancreas transplantation is the only method that can nearly cure insulin-dependent diabetes mellitus [1]. Despite rapid advances in islet transplant or artificial pancreas, pancreas transplantation outcomes remain superior [2,3]. While simultaneous pancreas and kidney transplantation, which is performed among patients with kidneys damaged mainly by diabetes mellitus, is used most widely, pancreas transplantation alone can be performed selectively for patients with type 1 diabetes mellitus with kidneys

Received September 6, 2019

Revised November 4, 2019

Accepted November 13, 2019

Correspondence to: Byung Hyun Choi

Department of Surgery, Pusan National University Yangsan Hospital, 20 Geumo-ro, Mulgeum-eup, Yangsan 50612, Korea

Tel: +82-55-360-2478, Fax: +82-55-360-2154

E-mail:gmoolpop@gmail.com that function well [4]. Successful pancreas transplantation without any complications, such as transplant rejection, helps to control glucose levels, and has a mostly positive effect on diabetic complications [1]. Nevertheless, the effect of pancreas transplantation on diabetic nephropathy remains controversial because immunosuppressants, such as calcineurin inhibitors, show nephrotoxicity $[5,6]$. Hence, pancreas transplantation alone is recommended when the glomerular filtration rate (GFR) is good ( $\geq 70$ $80 \mathrm{~mL} / \mathrm{min}$ ) and proteinuria is mild (within $1 \mathrm{~g} /$ day) [4]. In this report, we present the case of a patient who showed progression of diabetic nephropathy despite well-controlled glucose levels and lack of evidence of nephrotoxicity, caused by immunosuppressants, in the renal biopsy posttransplantation was found as the abovementioned conditions were met.

This is an Open Access article distributed under the terms of the Creative Commons Attribution Non-Commercial License (http://creativecommons. org/ licenses/by-nc/4.0/) which permits unrestricted non-commercial use, distribution, and reproduction in any medium, provided the original work is properly cited. 


\section{HIGHLIGHTS}

- Pancreas transplantation is the only method that can nearly cure insulin-dependent diabetes.

- Progression of diabetic nephropathy was observed with well controlled glucose level and no evidence of nephrotoxicity by immunosuppressants, after pancreas transplantation alone successfully.

\section{CASE REPORT}

The recipient was a 22-year-old female patient with no systemic conditions, such as hypertension and dyslipidemia, except diabetes mellitus. She was diagnosed with type 1 diabetes mellitus in another hospital 10 years prior at age 12. When she first visited our hospital as an outpatient, her glycated hemoglobin level was $11.1 \%$, which indicated poor glucose control. Forty units of insulin were administered daily, and she received laser treatment several times owing to diabetic retinopathy. Although her blood urea nitrogen and creatinine levels were normal ( 16.5 and $0.65 \mathrm{mg} / \mathrm{dL}$, respectively), the Chronic Kidney Disease Epidemiology Collaboration GFR was 123.3 $\mathrm{mL} / \mathrm{min} / 1.73 \mathrm{~m}^{2}$, and proteinuria (microalbumin, 253 $\mu \mathrm{g} / \mathrm{mL}$; albumin-to-creatinine ratio, $177.4 \mathrm{mg} / \mathrm{gm}$ ) was seen, which indicated that diabetic nephropathy had already developed. However, prior to transplantation, she had not taken any drug that was effective against proteinuria, such as angiotensin II receptor blockers or angiotensin-converting enzyme inhibitors (ACEi). Renal ultrasonography or biopsy was not conducted to evaluate her pretransplant renal function.

The pancreas was acquired from a 31-year-old woman who had become brain-dead owing to an ischemic brain injury. In the bench procedure, the donor's iliac artery bifurcation was used as the Y-vascular graft to reconstruct the superior mesenteric and splenic arteries of the transplanted pancreas. Some portion of the inferior vena cava acquired from the brain-dead donor was anastomosed to the portal vein of the transplanted pancreas in a diamond shape to prevent any stricture at the anastomosed site (Fig. 1) [7].

The recipient's abdominal cavity was approached through the mid-line, and the extended-Kocher maneuver, which separates the right colon and small intestine from the retroperitoneum, was used to move the intestines. Thereafter, the inferior vena cava and right common iliac artery were dissected. The portal vein of the transplanted pancreas was anastomosed to the inferior vena cava, and the Y-vascular graft was anastomosed to the right iliac artery. Subsequently, blood was reperfused to the transplanted pancreas. After hemostasis, the duodenum of the graft was directly anastomosed to the recipient's duodenum, which was intended for direct observation of the transplanted duodenum on endoscopy [7]. The drainage tube was inserted, the surgical wound was sutured, and the operation was completed.

Her glucose level was well-controlled right after the operation, and she was discharged around 3 postoperative weeks later without particular problems. Endoscopy was performed at 2 weeks postoperatively to observe the transplanted duodenum. A biopsy was also performed to verify that transplant rejection did not occur in the transplanted duodenum.

Thymoglobulin (anti-thymocyte globulin) was used as immune tolerance induction therapy and administered from the date of surgery to 2 days after surgery, on postoperative days 4 and 6 (five times in total). A total

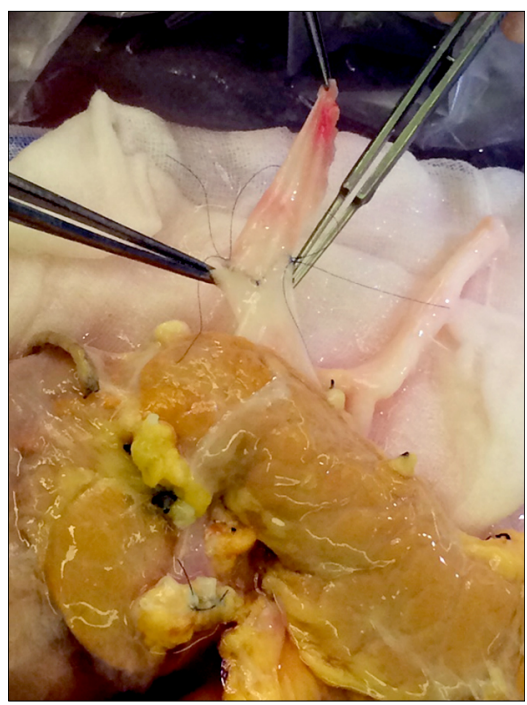

Fig. 1. Diamond-shaped patch is applied to the graft portal vein. Graft pancreas is soft with no fatty change. 


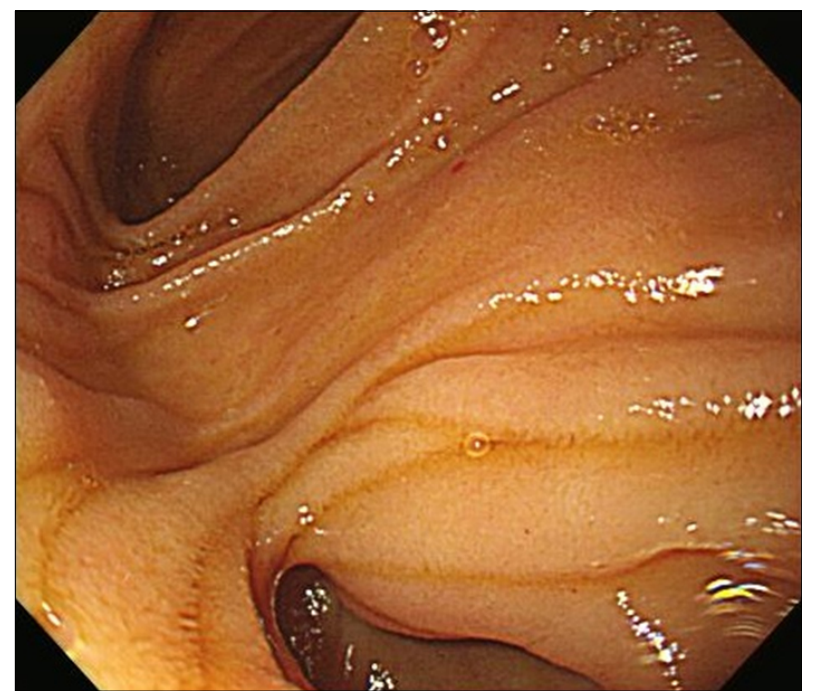

Fig. 2. Endoscopic finding of graft duodenum at postoperative 1 year. Heathy mucosa is observed. There is no evidence of rejection on duodenal mucosal biopsy. dose of $5 \mathrm{mg} / \mathrm{kg}$ was administered. Tacrolimus and mycophenolate mofetil were administered as immunosuppressants. The initial tacrolimus target range was 9$11 \mathrm{ng} / \mathrm{mL}$. The steroid dose was gradually decreased and discontinued in outpatient care after 2 postoperative months; only dual therapy was maintained. The outpatient follow-up continued without any noticeable complications (Fig. 2), and approximately 1 year after surgery, she was hospitalized and treated for Pneumocystis jiroveci pneumonia, which was cured. During the follow-up period, her glucose level was well-controlled (Fig. 3A), and no evidence of transplanted pancreas rejection was observed.

Approximately 2 months postoperatively, her creatinine levels increased (Fig. 3B), and we observed an increase in her proteinuria on development of an upper respiratory infection along with a decrease when the infection subsided (Fig. 3C). As she was infected with $P$.

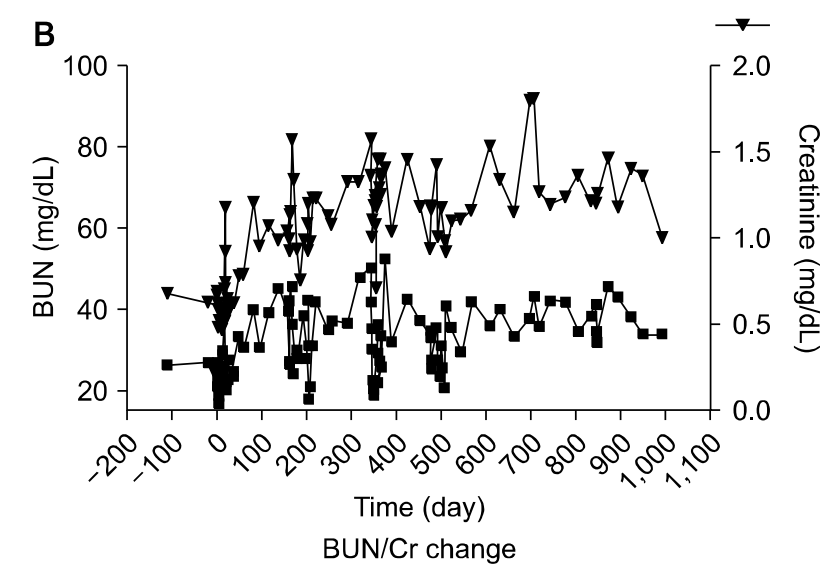

Fig. 3. (A) Glycosylated hemoglobin (HbA1c) level rapidly decreased to the normal range $(<6 \%)$ immediately after pancreas transplant alone. Fasting glucose level was also well controlled after transplant. (B) Blood urea nitrogen (BUN) and creatinine (Cr) levels fluctuating after transplant. Progression of diabetic nephropathy and use of immunosuppressant might deteriorate renal function. (C) Changes in posttransplant proteinuria. Proteinuria abruptly increases with three peaks, corresponding to Pneumocystis jiroveci pneumonia (PJP) infection, at postoperative 600 days and 800 days. 
jiroveci pneumonia around 1 year after the operation, her proteinuria had increased sharply. After recovery, it improved slightly. However, 17 months after the operation, her proteinuria began to increase again. As no decrease in proteinuria was observed despite high dose of angiotensin II receptor blocker, a renal biopsy was performed at 52 months after the operation.

The renal biopsy revealed no evidence of toxicity caused by immunosuppressants and no pathological finding other than diabetic nephropathy. Totally, eight glomeruli were included in the collected tissue, and we identified prominent thickening of the glomerular basement membrane (GBM) and Kimmelstiel-Wilson nodule on hematoxylin and eosin stain, which were strongly suggestive of diabetic nephropathy (Fig. 4). In addition, on electron microscopy, the GBM measured more than $500 \mathrm{~nm}$ in thickness (Fig. 5). Immunofluorescence staining showed a pattern of immunoglobulin G ( $\lg G$ ) staining along the GBM (Fig. 6). Based on these findings, the patient was diagnosed with diabetic nephropathy. Global sclerosis was observed in 3 glomeruli $(3 / 8,37.5 \%)$ and thickening of the capillary wall in 5 glomeruli. The renal tubule was normal, and mild fibrosis was observed in the interstitium. Arteriosclerosis was observed in the blood vessels. Up to 4 months after diagnosis, angiotensin II receptor blockers and diuretics were used. Currently, her creatinine finding did not worsen, but proteinuria showed no improvement. Despite increase in proteinuria after transplant, her blood pressure was always within the normal range $(\leq 130 / 80 \mathrm{mmHg})$. No hematuria was observed during the entire period.

As retinal edema occurred after pancreas transplant, the patient received an Avastin intravitreal injection 2 months and 2 years after the transplant, and a binocular retinal laser treatment around a similar time (YAG laser). The patient underwent binocular cataract surgery
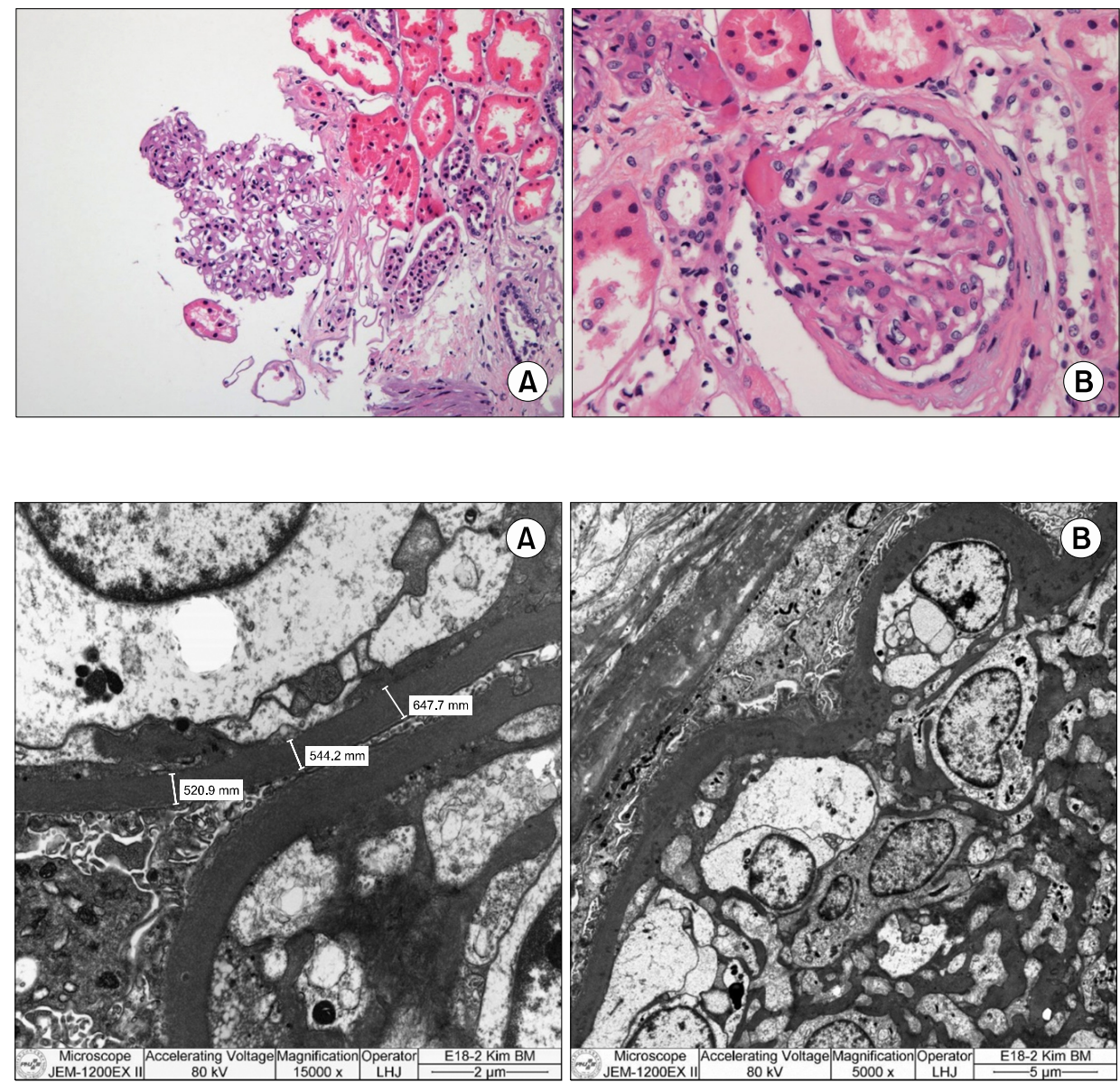

Fig. 4. (A, B) Pathologic findings of renal biopsy. Prominent glomerular basement membrane thickening is identified. Deposits of hyaline material are present under the parietal epithelium of Bowman capsule, forming Kimmelstiel- Wilson nodule in one glomerulus. Global sclerosis is identified (H\&E; A: $\times 200, \quad B$ $\times 400$ ).

Fig. 5. (A, B) Pathologic findings of renal biopsy. On electron microscopy, foot process effacement is diffuse. Glomerular basement membrane is thick, measuring more than $500 \mathrm{~nm}$ in thickness. Mesangial sclerosis is identified. Electron dense deposit is not identified. 


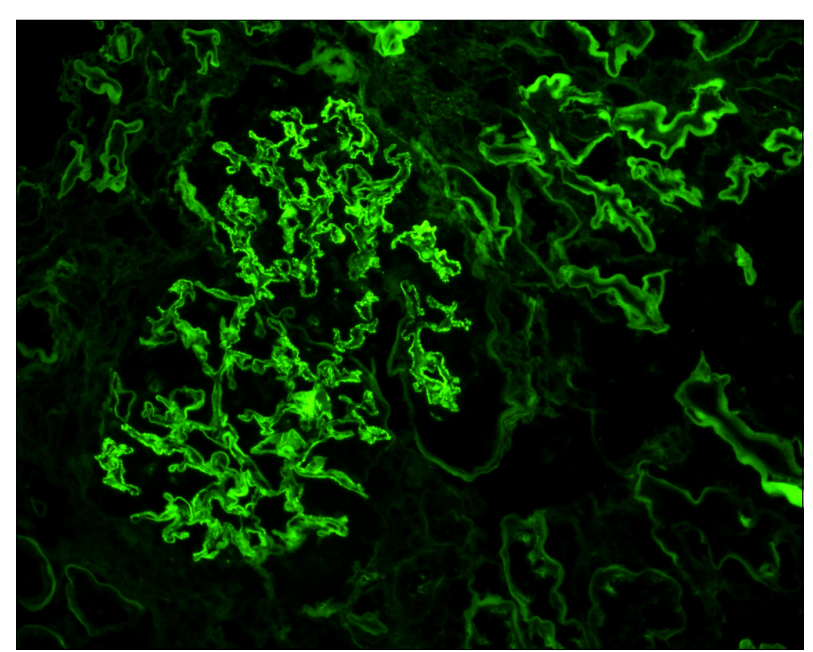

Fig. 6. Linear immunofluorescence stain of immunoglobulin G shows along the glomerular basement membrane.

for corneal opacity around 25 months after surgery. In the past 1 year, she maintained a visual acuity of 0.1 in the right eye and 0.3 in the left eye. While her diabetic retinopathy is not progressing further, she needs continued monitoring of its progression.

\section{DISCUSSION}

In 1998, Fioretto et al. [8] reported that the glucose levels were maintained at normal for a long time after pancreas transplant alone; however, the GFR of the recipients who received a pancreas transplant may decrease slightly owing to immunosuppressants. Nevertheless, the patients showed histological improvements, their proteinuria decreased, the thickness of the glomerular and renal tubular basement membranes decreased in the biopsy, and the size of the mesangium decreased. In 2011, a report indicated that proteinuria improved substantially although GFR decreased after pancreas transplantation alone [9]. Given these results, one may think that if a pancreas transplant is performed in the patient with uncontrolled glucose levels before transplant, it would improve diabetic nephropathy and have a greater protective effect on the kidney. Another study by Fioretto et al. [10] in 2014, showed that diabetic nephropathy could be reversible through pancreatic transplantation alone; however, during the first 5 years of follow-up, no change was seen in the existing kidney lesions, and it took 10 years to be normalized with long-term of normoglycemia [11]. Thus, even if no change in diabetic kidney disease is identified immediately after transplantation, strict glucose level control and long-term follow-up are warranted, owing to the possibility of reversal to normal kidney structure.

However, owing to the high risk of nephrotoxicity due to immunosuppressants, no conclusion has been drawn regarding the decline in the risk of progression into terminal nephropathy for transplant recipient following pancreas transplant owing to well-controlled glucose levels [5,12].

A long-term observation of progression from type 1 diabetes into diabetic nephropathy shows that GFR increases initially and then decreases rapidly after a certain period [13]. If a patient has only microalbuminuria and normal GFR, improvements into a reversible condition can be expected with active treatment. If macroalbuminuria is present and the GFR begins to decline, the patient's diabetic nephropathy is highly likely to have progressed and become irreversible already [14]. Thereafter, the treatment goal becomes to delay the disease progression. Diabetic nephropathy is prevented or treated by controlling the glucose level, blood pressure, and blood lipid level, and drug therapy, including ACEi or angiotensin II receptor blockers, are known to reduce proteinuria [15]. However, although this patient had normal GFR, showed no dyslipidemia, and controlled her glucose level well after pancreas transplant, her creatinine level increased slightly, while her proteinuria increased gradually, except increase and recovery sharply during systemic infection. We believe that nephrotoxicity arising from immunosuppressants may have influenced it. Given that her proteinuria increased rapidly when her systemic condition, such as infections deteriorated, diabetic nephropathy at the time of transplant may have progressed further than that identified by the creatinine level or proteinuria.

For the treatment of diabetic retinopathy or retinal edema, intravitreal injection of vascular endothelial growth factor inhibitors has been used; however, it is pointed out that bevacizumab may exacerbate proteinuria and kidney 
disease [16,17]. However, intravitreal injection is more than 150 times lower than the systemic dosage, and it is reported that it is hard to confirm the clinical correlation [16]. However, progression and recurrence of minimal change disease of the kidney, and progression to renal failure was reported in severe cases $[18,19]$. Thus, caution must be exercised to treat diabetic retinopathy or edema with chronic kidney disease.

In determining the condition of chronic nephropathy, including diabetic nephropathy, it would be difficult to accurately identify the condition of the kidney on the basis of creatinine level or GFR alone, since currently used immunosuppressive agents, especially calcineurin inhibitor, have important renal effects, decreasing both GFR and albumin excretion rate [13].

In conclusion, when pancreas transplant alone is performed, accurate identification of the recipient's renal function and proteinuria is required. If possible, transplantation is recommended before progression to irreversible diabetic nephropathy. If the renal function deteriorates or proteinuria increases after transplant, it would be important not only to conclude that it has been caused by immunosuppressants but also to consider the possibility that diabetic nephropathy has progressed despite well-controlled glucose levels and manage the condition accordingly.

\section{ACKNOWLEDGMENTS}

\section{Conflict of Interest}

No potential conflict of interest relevant to this article was reported.

\section{Funding/Support}

This study was supported by research grant from the Korean Society for Transplantation (2019-04-02001010).

\section{ORCID}

Yoonhong Kim

Dong Il Kim

Jae Ryong Shim

Tae Beom Lee https://orcid. org/0000-0003-1611-5390 https://orcid. org/0000-0001-9874-1322 https://orcid. org/0000-0002-9032-9373 https://orcid. org/0000-0002-9603-965X
Kwang Ho Yang

https://orcid. org/0000-0003-1489-6787

Je Ho Ryu https://orcid. org/0000-0002-9377-3074

Hyun Jung Lee https://orcid. org/0000-0002-2995-6060

Byung Hyun Choi https://orcid. org/0000-0002-9245-5066

\section{Author Contributions}

Conceptualization: YK, BHC. Data curation: YK, DIK, JRS, TBL, KHY, JHR. Formal analysis: YK, DIK, JRS, TBL, KHY, JHR, HJL. Funding acquisition: BHC. Methodology: YK, HJL, BHC. Project administration: BHC. Visualization: YK, BHC. Writing - original draft: YK, BHC. Writing - review \& editing: YK, BHC.

\section{REFERENCES}

1. Dean PG, Kukla A, Stegall MD, Kudva YC. Pancreas transplantation. BMJ 2017;357:j1321.

2. Wisel SA, Braun HJ, Stock PG. Current outcomes in islet versus solid organ pancreas transplant for $\beta$-cell replacement in type 1 diabetes. Curr Opin Organ Transplant 2016; 21:399-404.

3. Bekiari E, Kitsios $\mathrm{K}$, Thabit $\mathrm{H}$, Tauschmann $\mathrm{M}$, Athanasiadou E, Karagiannis T, et al. Artificial pancreas treatment for outpatients with type 1 diabetes: systematic review and meta-analysis. BMJ 2018;361:k1310.

4. Redfield RR, Rickels MR, Naji A, Odorico JS. Pancreas transplantation in the modern era. Gastroenterol Clin North Am 2016;45:145-66.

5. Shin S, Jung CH, Choi JY, Kwon HW, Jung JH, Kim YH, et al. Long-term effects of pancreas transplant alone on nephropathy in type 1 diabetic patients with optimal renal function. PLoS One 2018;13:e191421.

6. Ruospo M, Saglimbene VM, Palmer SC, De Cosmo S, Pacilli A, Lamacchia O, et al. Glucose targets for preventing diabetic kidney disease and its progression. Cochrane Database Syst Rev 2017;6:CD010137.

7. Ryu JH, Lee TB, Park YM, Yang KH, Chu CW, Lee JH, et al. Pancreas transplant with duodeno-duodenostomy and caval drainage using a diamond patch graft: a singlecenter experience. Ann Transplant 2017;22:24-34.

8. Fioretto P, Steffes MW, Sutherland DE, Goetz FC, Mauer M. Reversal of lesions of diabetic nephropathy after pancreas transplantation. N Engl J Med 1998;339:69-75.

9. Boggi U, Vistoli F, Amorese G, Giannarelli R, Coppelli A, Mariotti R, et al. Results of pancreas transplantation alone with special attention to native kidney function and proteinuria in type 1 diabetes patients. Rev Diabet Stud 


\section{1;8:259-67.}

10. Fioretto P, Barzon I, Mauer M. Is diabetic nephropathy reversible? Diabetes Res Clin Pract 2014;104:323-8.

11. Mauer M, Fioretto P. Pancreas transplantation and reversal of diabetic nephropathy lesions. Med Clin North Am 2013;97:109-14.

12. Singh SK, Kim SJ, Smail N, Schiff J, Paraskevas S, Cantarovich M. Outcomes of recipients with pancreas transplant alone who develop end-stage renal disease. Am J Transplant 2016;16:535-40.

13. Maahs DM. Early detection of kidney disease in type 1 diabetes: what do we really know? Diabetes Technol Ther 2012;14:541-4.

14. Bjornstad P, Cherney D, Maahs DM. Early diabetic nephropathy in type 1 diabetes: new insights. Curr Opin Endocrinol Diabetes Obes 2014;21:279-86.

15. Lin YC, Chang YH, Yang SY, Wu KD, Chu TS. Update of pathophysiology and management of diabetic kidney disease. J Formos Med Assoc 2018;117:662-75.

16. Kameda Y, Babazono T, Uchigata Y, Kitano S. Renal function after intravitreal administration of vascular endothelial growth factor inhibitors in patients with diabetes and chronic kidney disease. J Diabetes Investig 2018;9: 937-9.

17. Lafayette RA, McCall B, Li N, Chu L, Werner P, Das $A$, et al. Incidence and relevance of proteinuria in bevacizumab-treated patients: pooled analysis from randomized controlled trials. Am J Nephrol 2014;40:75-83.

18. Hanna RM, Lopez E, Wilson J, Barathan S, Cohen AH. Minimal change disease onset observed after bevacizumab administration. Clin Kidney J 2016;9:239-44.

19. Pérez-Valdivia MA, López-Mendoza M, Toro-Prieto FJ, Cabello-Chaves V, Toro-Ramos M, Martín-Herrera MC, et al. Relapse of minimal change disease nephrotic syndrome after administering intravitreal bevacizumab. Nefrologia 2014;34:421-2. 